\title{
COXETER GROUPS ARE NOT HIGHER RANK ARITHMETIC GROUPS
}

\author{
SANDIP SINGH
}

\begin{abstract}
Let $\mathrm{W}$ be an irreducible finitely generated Coxeter group. The geometric representation of $\mathrm{W}$ in $\mathrm{GL}(\mathrm{V})$ provides a discrete embedding in the orthogonal group of the Tits form (the associated bilinear form of the Coxeter group). If the Tits form of the Coxeter group is non-positive and non-degenerate, the Coxeter group does not contain any finite index subgroup isomorphic to an irreducible lattice in a semisimple group of $\mathbb{R}$-rank $\geq 2$.
\end{abstract}

\section{Introduction}

Let $S=\left\{s_{1}, s_{2}, \ldots, s_{n}\right\}$ be a finite set and $\mathrm{W}$ be a group generated by $S$ with the relations

$$
\left(s_{i} s_{j}\right)^{m_{i, j}}=1,
$$

where $m_{i, i}=1, \forall 1 \leq i \leq n$ and $m_{i, j} \in\{2,3, \ldots, \infty\}, \forall i \neq j$. The group $\mathrm{W}$ is called the Coxeter group. The Coxeter system $(\mathrm{W}, S)$ is called irreducible if the Coxeter graph ([4, Section 2.1]) is connected. Now we define a symmetric bilinear form (Tits form) $B$ on a vector space $\mathrm{V}$ of $\operatorname{dim} n$ over $\mathbb{R}$, with a basis $\left\{e_{1}, e_{2}, \ldots, e_{n}\right\}$ in one-to-one correspondence with $S$ as

$$
B\left(e_{i}, e_{j}\right)=-\cos \left(\frac{\pi}{m_{i, j}}\right), \quad \forall 1 \leq i, j \leq n .
$$

(This expression is interpreted to be -1 in case $m_{i, j}=\infty$.)

For each $s_{i} \in S$, we can now define a reflection $\sigma_{i}: \mathrm{V} \rightarrow \mathrm{V}$ by the rule:

$$
\sigma_{i} \lambda=\lambda-2 B\left(e_{i}, \lambda\right) e_{i} .
$$

Clearly $\sigma_{i} e_{i}=-e_{i}$, while $\sigma_{i}$ fixes $H_{i}=\left\{v \in \mathrm{V} \mid B\left(v, e_{i}\right)=0\right\}$ pointwise. In particular, we see that $\sigma_{i}$ has order 2 in $\operatorname{GL}(\mathrm{V})$. The bilinear form $B$ is preserved by all of the elements $\sigma_{i}$, and hence it will be preserved by each element of the subgroup of GL(V) generated by the $\sigma_{i}(1 \leq i \leq n)$.

By defining $s_{i} \mapsto \sigma_{i}$, we get a unique homomorphism $\sigma: \mathrm{W} \rightarrow \mathrm{GL}(\mathrm{V})$ sending $s_{i}$ to $\sigma_{i}$, and the group $\sigma(\mathrm{W})$ preserves the form $B$ on $\mathrm{V}$; and for each pair $s_{i}, s_{j} \in S$, the order of $s_{i} s_{j}$ in $\mathrm{W}$ is precisely $m_{i, j}([4$, Proposition 5.3]). Also, the representation $\sigma: \mathrm{W} \rightarrow \mathrm{GL}(\mathrm{V})$ is faithful ([4, Corollary 5.4]).

Relative to the basis $\left\{e_{1}, e_{2}, \ldots, e_{n}\right\}$ of $\mathrm{V}$, we can identify $\mathrm{V}$ with $\mathbb{R}^{n}$ and $\mathrm{GL}(\mathrm{V})$ with $\operatorname{GL}(n, \mathbb{R})$, the latter in turn being viewed as an open set in $\mathbb{R}^{n^{2}}$. It follows from $[4$, Proposition 6.2] that $\sigma(\mathrm{W})$ is a discrete subgroup of $\mathrm{GL}(\mathrm{V})$.

Received by the editors January 9, 2012.

2010 Mathematics Subject Classification. Primary: 20F55; Secondary: 22E40.

Key words and phrases. Coxeter groups, Irreducible lattices, Orthogonal groups, Superrigidity. 
In this paper, we will assume that the Coxeter system $(\mathrm{W}, S)$ is irreducible and the Tits form $B$ is non-degenerate and the Coxeter group $\mathrm{W}$ is infinite. By the earlier observations, it follows that $\mathrm{W}$ is a discrete subgroup of the corresponding orthogonal group $\mathrm{G}:=\mathrm{O}(B)(\mathbb{R})$. Moreover, $\mathrm{G}$ is a real Lie group, with a Haar measure, which provides a notion of volume $\nu$ for $\mathrm{W} \backslash \mathrm{G}$, the homogeneous space of right cosets of $\mathrm{G}$ with respect to $\mathrm{W}$. If the measure $\nu$ on $\mathrm{W} \backslash \mathrm{G}$ is finite and G-invariant, then $\mathrm{W}$ is a lattice in G.

The goal of this paper is to prove Theorem 1.1 (stated below), which has been proved in [3] also, by using a different technique. They have proved that an infinite Coxeter group has a subgroup of finite index which admits a homomorphism onto $\mathbb{Z}([3$, Theorem 1.1]) and used it to prove the theorem. I have tried here to give an elementary proof of the theorem by using a Bourbaki exercise (Para 12, Exercise Section 4 of Chapter V in [2]) and Margulis superrigidity (Theorem 1.4, below).

Theorem 1.1. If $\mathrm{W}$ is an irreducible finitely generated Coxeter group with the nonpositive and non-degenerate Tits form, then it does not contain any finite index subgroup isomorphic to an irreducible lattice in a connected semisimple Lie group without non-trivial compact factor groups, of real rank $\geq 2$.

In fact more is true:

Theorem 1.2. (a) If $\mathrm{W}$ is an irreducible finitely generated Coxeter group with the non-positive and non-degenerate Tits form, then it does not contain any finite index subgroup isomorphic to a higher rank S-arithmetic group (i.e., lattice in a product of Lie groups and p-adic groups).

For example, the Coxeter group $\mathrm{W}$ does not contain any finite index subgroup isomorphic to $\mathrm{SL}_{2}\left(\mathbb{Z}\left[\frac{1}{p}\right]\right)$ in $\mathrm{SL}_{2}(\mathbb{R}) \times \mathrm{SL}_{2}\left(\mathbb{Q}_{p}\right)$.

(b) More generally, if $k_{1}, k_{2}, \ldots, k_{r}$ are local fields and $\mathrm{G}_{1}, \mathrm{G}_{2}, \ldots, \mathrm{G}_{r}$ are semisimple algebraic groups defined over $k_{1}, k_{2}, \ldots, k_{r}$ respectively such that each $\mathrm{G}_{i}$ has $k_{i}$-rank $\geq 1$ and $\sum_{i=1}^{r} k_{i}$-rank $\left(\mathrm{G}_{i}\right) \geq 2$, then $\mathrm{W}$ does not contain any finite index subgroup isomorphic to an irreducible lattice $\Gamma$ in $\prod_{i=1}^{r} \mathrm{G}_{i}\left(k_{i}\right)$.

For example, the Coxeter group $\mathrm{W}$ does not contain any finite index subgroup isomorphic to $\mathrm{SL}_{3}\left(\mathbb{F}_{p}[t]\right)$ in $\mathrm{SL}_{3}\left(\mathbb{F}_{p}\left(\left(\frac{1}{t}\right)\right)\right)$.

Theorem 1.2 can be proved by the same method used for the proof of Theorem 1.1 using Theorem 1.3 (stated below) and the superrigidity of lattices in semisimple groups over local fields of arbitrary characteristic (see [6]; cf. [9]). Therefore, in this paper we will prove Theorem 1.1; and for the sake of completeness of the proof we will also prove the following theorem (stated in [2] as an exercise):

Theorem 1.3 (Para 12, Exercise Section 4 of Chapter $\mathrm{V}$ in [2]). If $\mathrm{W}$ is a lattice in $\mathrm{O}(B)(\mathbb{R})$, then $B$ has signature $(n-1,1)$ and $B(v, v)<0$, for all $v \in \mathrm{C}$, where $\mathrm{C}:=\left\{v \in \mathrm{V} \mid B\left(v, e_{i}\right)>0, \forall 1 \leq i \leq n\right\}$.

Note that a Coxeter group $\mathrm{W}$ can not be a lattice in $\mathrm{O}(B)(\mathbb{R})=\mathrm{O}(n-1,1)$, for $n>10$ (17, Exercise Section 4 of Chapter V in [2]).

To prove Theorem 1.1 we will use the following theorem of G. A. Margulis:

Theorem 1.4 (Theorem 6.16 of Chapter IX in [6]). Let $\mathrm{H}$ be a connected semisimple Lie group without non-trivial compact factor groups. Let $\Gamma \subset \mathrm{H}$ be a lattice, $k$ a 
local field, $\mathrm{F}$ a connected semisimple $k$-group, and $\delta: \Gamma \longrightarrow \mathrm{F}(k)$ a homomorphism such that the subgroup $\delta(\Gamma)$ is Zariski dense in $\mathrm{F}$. Assume that rank $\mathrm{H} \geq 2$ and the lattice $\Gamma$ is irreducible. Then,

(a) for $k$ isomorphic neither to $\mathbb{R}$ nor to $\mathbb{C}$, i.e., for non-archimedean $k$, the subgroup $\delta(\Gamma)$ is relatively compact in $\mathrm{F}(k)$.

(b) for $k=\mathbb{R}$, if the group $\mathrm{F}$ is adjoint and has no non-trivial $\mathbb{R}$ - anisotropic factors, then $\delta$ extends, uniquely, to a continuous homomorphism $\tilde{\delta}: \mathrm{H} \longrightarrow$ $\mathrm{F}(\mathbb{R})$.

In this paper (Section 4), we will also show that a right-angled Coxeter group W generated by three elements is isomorphic to a lattice in the group $\mathrm{O}(B)(\mathbb{R})=\mathrm{O}(2,1)$ of real rank 1.

\section{Proof of Theorem 1.3}

The proof has been sketched in the Bourbaki exercise (Para 12, Exercise Section 4 of Chapter $\mathrm{V}$ in [2]), and for the sake of completeness we fill in the details.

If $s_{i} \in S$, denote by $\mathrm{A}_{\mathrm{i}}$, the set of $x \in \mathrm{V}$ such that $B\left(x, e_{i}\right)>0$. Clearly $\mathrm{C}=\cap_{i=1}^{n} \mathrm{~A}_{i}$ is an open set in $\mathrm{V}$, if $S$ is finite. The following theorem is from [2]:

Theorem 2.1 (Tits). If $w \in \mathrm{W}$ and $\mathrm{C} \cap w \mathrm{C} \neq \emptyset$, then $w=1$.

Let $\mathrm{G}$ be a closed subgroup of $\mathrm{GL}(\mathrm{V})$ containing $\mathrm{W}$. Let $\mathrm{G}$ be unimodular and $\mathrm{D}$ be a half line of $\mathrm{V}$ contained in $\mathrm{C}$, i.e., $\mathrm{D}=\mathbb{R}_{>0} v \subset \mathrm{C}$, for some $v \in \mathrm{C}$, and let $\mathrm{G}_{\mathrm{D}}$ be the stabilizer of $\mathrm{D}$ in $\mathrm{G}$. With these notation, we get the following lemma:

Lemma 2.2. Let $\Delta$ be the set of elements $g \in \mathrm{G}$ such that $g(\mathrm{D}) \subset \mathrm{C}$. Then $\Delta$ is open, stable under right multiplication by $\mathrm{G}_{\mathrm{D}}$, and that the composite map $\Delta \longrightarrow$ $\mathrm{G} \longrightarrow \mathrm{W} \backslash \mathrm{G}$ is injective, where $\mathrm{W} \backslash \mathrm{G}$ denotes the homogeneous space of right cosets of $\mathrm{G}$ with respect to $\mathrm{W}$.

Proof. First, we show that $\Delta$ is open in G. For, $\Delta=\{g \in \mathrm{G} \mid g(v) \in \mathrm{C}\}$, where $v \in \mathrm{V}$ such that $\mathrm{D}=\mathbb{R}_{>0} v \subset \mathrm{C}$. We define a map $f: \mathrm{G} \longrightarrow \mathrm{V}$ by $g \mapsto g(v)$. It is clear that $f$ is continuous and $\mathrm{C}$ is open in $\mathrm{V}$, hence $f^{-1}(\mathrm{C})=\Delta$ is open in $\mathrm{G}$.

Now we show that $\Delta$ is stable under right multiplication by $\mathrm{G}_{\mathrm{D}}$. For, let $h \in \mathrm{G}_{\mathrm{D}}$ and $g \in \Delta$. Then

$$
g h(v)=g(\alpha v)=\alpha g(v) \in \mathrm{C}, \text { for some } \alpha \in \mathbb{R}_{>0},
$$

and this shows that $g h \in \Delta$.

Finally, we show that the composite map $\Delta \longrightarrow \mathrm{G} \longrightarrow \mathrm{W} \backslash \mathrm{G}$ is injective. For, let $g_{1}, g_{2} \in \Delta$ such that $\mathrm{W} g_{1}=\mathrm{W} g_{2}$, i.e., $g_{1} g_{2}^{-1} \in \mathrm{W}$. Since $g_{2}(\mathrm{D}) \subset \mathrm{C}, \mathrm{D} \subset g_{2}^{-1}(\mathrm{C})$. That is, $g_{1}(\mathrm{D}) \subset g_{1} g_{2}^{-1}(\mathrm{C})$. Also, $g_{1}(\mathrm{D}) \subset \mathrm{C}$, therefore $g_{1} g_{2}^{-1}(\mathrm{C}) \cap \mathrm{C} \neq \emptyset$. Hence by Theorem 2.1 , we get $g_{1} g_{2}^{-1}=1$. This shows that the composite map $\Delta \longrightarrow \mathrm{G} \longrightarrow$ $\mathrm{W} \backslash \mathrm{G}$ is injective.

Lemma 2.3. Let $\mu$ be a Haar measure on $\mathrm{G}$. If $\mu(\Delta)$ is finite, the subgroup $\mathrm{G}_{\mathrm{D}}$ is compact. 
Proof. Since $\Delta$ is an open set containing the identity element of $\mathrm{G}$ and the group $\mathrm{G}$ is locally compact, there exists a compact neighbourhood $\mathrm{K}$ of the identity element contained in $\Delta$.

We now claim that there exist finitely many elements $h_{i} \in \mathrm{G}_{\mathrm{D}}$ such that every set of the form $\mathrm{K} h$, with $h \in \mathrm{G}_{\mathrm{D}}$, meets one of the $\mathrm{K} h_{i}$. For, suppose on the contrary that $\forall k \in \mathbb{N}$ and $\mathcal{H}_{k}=\left\{h_{1}, h_{2}, \ldots, h_{k}\right\}$ collection of elements in $\mathrm{G}_{\mathrm{D}}$, there exists $h_{k+1} \in \mathrm{G}_{\mathrm{D}}$ such that $\mathrm{K} h_{k+1} \cap\left(\cup_{i=1}^{k} \mathrm{~K} h_{i}\right)=\emptyset$. It is also clear that $\mathrm{K} h_{i} \cap \mathrm{K} h_{j}=\emptyset, \forall i \neq j$.

Since $\Delta$ is stable under right multiplication by any element of $\mathrm{G}_{\mathrm{D}}$, we get $\mathrm{K} h \subset$ $\Delta, \forall h \in \mathrm{G}_{\mathrm{D}}$. Hence

$$
\mu(\Delta) \geq \mu\left(\cup_{i=1}^{\infty} \mathrm{K} h_{i}\right)=\sum_{i=1}^{\infty} \mu\left(\mathrm{K} h_{i}\right)=\sum_{i=1}^{\infty} \mu(\mathrm{K})=\infty
$$

(since $\mathrm{G}$ is unimodular and $\mathrm{K}$ contains an open subset of $\mathrm{G}, \mu(\mathrm{K})>0$ ), which is a contradiction to the given hypothesis. Therefore, $\exists \mathcal{H}_{r}=\left\{h_{1}, h_{2}, \ldots, h_{r}\right\}$ a finite collection of elements in $\mathrm{G}_{\mathrm{D}}$ such that $\forall h \in \mathrm{G}_{\mathrm{D}}, \mathrm{K} h \cap \mathrm{K} h_{i} \neq \emptyset$, for some $i \in\{1,2, \ldots, r\}$, which shows that $\mathrm{G}_{\mathrm{D}} \subset \cup_{i=1}^{r} \mathrm{~K}^{-1} \mathrm{~K} h_{i}$ and hence $\mathrm{G}_{\mathrm{D}}$ is compact (since $\mathrm{G}_{\mathrm{D}}$ is a closed subset of $\mathrm{G}$ and $\cup_{i=1}^{r} \mathrm{~K}^{-1} \mathrm{~K} h_{i}$ is compact).

Lemma 2.4. Let $\nu$ be a non-zero positive measure on $\mathrm{W} \backslash \mathrm{G}$, invariant under $\mathrm{G}$. If $\nu(\mathrm{W} \backslash \mathrm{G})<\infty$, then $\mathrm{G}_{\mathrm{D}}$ is compact.

Proof. Recall that $\mathrm{G}$ is unimodular with a Haar measure $\mu$ and $\nu$ is a non-zero positive measure on $\mathrm{W} \backslash \mathrm{G}$, invariant under G. Let $\nu^{\prime}$ be a Haar measure on $\mathrm{W}$. Since $\mathrm{W}$ is a discrete subgroup of $\mathrm{GL}(V), \nu^{\prime}$ is actually the counting measure (up to a scalar multiple) on $\mathrm{W}$. We prove here that $\mu(\Delta)<\infty$, which proves that $\mathrm{G}_{\mathrm{D}}$ is compact, using the last lemma.

We have a relation in $\mu, \nu$ and $\nu^{\prime}$ as

$$
\int_{\mathrm{G}} f d \mu=\int_{\mathrm{W} \backslash \mathrm{G}}\left(\int_{\mathrm{W}} f(w g) d \nu^{\prime}(w)\right) d \nu(\mathrm{W} g), \forall f \in \mathcal{C}_{c}(\mathrm{G})
$$

where $\mathcal{C}_{c}(\mathrm{G})$ is the space of all compactly supported continuous functions on $\mathrm{G}$.

Let the symbol $f \prec \Delta$ means that $f \in \mathcal{C}_{c}(\mathrm{G})$ with $0 \leq f \leq 1$ and the support of $f$ is contained in $\Delta$. Since $\Delta$ is open in $\mathrm{G}$, we get

$$
\mu(\Delta)=\sup \left\{\int_{\mathrm{G}} f d \mu: f \prec \Delta\right\} .
$$

Let $f \prec \Delta$. By $(2.1)$, we get

$$
\begin{aligned}
\int_{\mathrm{G}} f d \mu & =\int_{\mathrm{W} \backslash \mathrm{G}}\left(\int_{\mathrm{W}} f(w g) d \nu^{\prime}(w)\right) d \nu(\mathrm{W} g) \\
& \leq \int_{\mathrm{W} \backslash \mathrm{G}}\left(\int_{\mathrm{W}} \chi_{\Delta}(w g) d \nu^{\prime}(w)\right) d \nu(\mathrm{W} g)
\end{aligned}
$$

where $\chi_{\Delta}$ is the characteristic function of $\Delta$. 
Since $w g \in \Delta \Leftrightarrow w \in \Delta g^{-1}$, we get

$$
\begin{aligned}
\int_{\mathrm{W} \backslash \mathrm{G}}\left(\int_{\mathrm{W}} \chi_{\Delta}(w g) d \nu^{\prime}(w)\right) d \nu(\mathrm{W} g) & =\int_{\mathrm{W} \backslash \mathrm{G}} \nu^{\prime}\left(\Delta g^{-1} \cap \mathrm{W}\right) d \nu(\mathrm{W} g) \\
& =\int_{\mathrm{W} \backslash \mathrm{G}} \#\left(\Delta g^{-1} \cap \mathrm{W}\right) d \nu(\mathrm{W} g),
\end{aligned}
$$

where $\#\left(\Delta g^{-1} \cap \mathrm{W}\right)$ denotes the number of elements in the set $\Delta g^{-1} \cap \mathrm{W}$.

Since $x \in \Delta g^{-1} \cap \mathrm{W} \Leftrightarrow x g \in \Delta$ and $x \in \mathrm{W}$, we get

$$
x g(\mathrm{D}) \subset \mathrm{C} \text { i.e. } x g(v) \in \mathrm{C}, \forall x \in \Delta g^{-1} \cap \mathrm{W}\left(\because \mathrm{D}=\mathbb{R}_{>0} v\right) .
$$

Now we claim that $\#\left(\Delta g^{-1} \cap \mathrm{W}\right) \leq 1$. For, let $x_{1}, x_{2} \in \Delta g^{-1} \cap \mathrm{W}$. Then, we get the following:

$$
\begin{aligned}
x_{1} g(v) & =c_{1} \in \mathrm{C} \quad \text { and } \quad x_{2} g(v)=c_{2} \in \mathrm{C} \\
& \Rightarrow x_{2} x_{1}^{-1}\left(c_{1}\right)=x_{2} x_{1}^{-1}\left(x_{1}(g v)\right)=x_{2}(g v)=c_{2} \\
& \Rightarrow x_{2} x_{1}^{-1}(\mathrm{C}) \cap \mathrm{C} \neq \emptyset \\
& \Rightarrow x_{2} x_{1}^{-1}=1 \quad \text { (by Theorem 2.1) } \\
& \Rightarrow x_{2}=x_{1} .
\end{aligned}
$$

Therefore, $\#\left(\Delta g^{-1} \cap \mathrm{W}\right) \leq 1$, and we get

$$
\begin{aligned}
\int_{\mathrm{W} \backslash \mathrm{G}} \#\left(\Delta g^{-1} \cap \mathrm{W}\right) d \nu(\mathrm{W} g) & \leq \int_{\mathrm{W} \backslash \mathrm{G}} d \nu(\mathrm{W} g) \\
& =\nu(\mathrm{W} \backslash \mathrm{G}) .
\end{aligned}
$$

By $(2.3)-(2.5)$, we get

$$
\int_{\mathrm{G}} f d \mu \leq \nu(\mathrm{W} \backslash \mathrm{G}) .
$$

As $f \prec \Delta$ was chosen arbitrarily, we get $\mu(\Delta) \leq \nu(\mathrm{W} \backslash \mathrm{G}$ ) (by using (2.2)), and hence $\mu(\Delta)<\infty$.

Now we prove Theorem 1.3 using the above lemmas. We have $B$, a non-degenerate bilinear form on $V$. Let $G$ be the group of real points of the orthogonal group of $B$ and $\mu$ be a Haar measure on $\mathrm{G}$. It is clear that the group $\mathrm{G}$ is unimodular and contains W. Since $\mathrm{W}$ is infinite, the bilinear form $B$ is not positive definite and it has the signature $(p, q)$, where $p+q=n$ and $p, q \geq 1$. We prove few more lemmas to prove Theorem 1.3 .

Lemma 2.5. $B(v, v) \neq 0$, for some $v \in \mathrm{C}$.

Proof. Since for any $v \in \mathrm{C}, \mathrm{C}-v$ is an open subset of $\mathrm{V}$ containing the origin 0 ( since $\mathrm{C}$ is an open subset of $\mathrm{V}$ ), $\mathrm{V}$ is generated by $\mathrm{C}-v$ (as an abelian group). In particular, $\mathrm{C}-v$ generates $\mathrm{V}$ as a vector space over $\mathbb{R}$, therefore there exists $\left\{v_{1}-v, v_{2}-v, \ldots, v_{n}-v\right\}$ a basis of $\mathrm{V}$ over $\mathbb{R}$ contained in $\mathrm{C}-v$, where $v_{i} \in \mathrm{C}, \forall 1 \leq$ $i \leq n$. Now if possible, let $B(v, v)=0, \forall v \in \mathrm{C}$.

$$
\begin{aligned}
\Rightarrow B(u, v) & =\frac{1}{2}(B(u+v, u+v)-B(u, u)-B(v, v)) \\
& =0 \quad \forall u, v \in \mathrm{C} \quad(\because \forall u, v \in \mathrm{C}, u+v \in \mathrm{C}) .
\end{aligned}
$$


Now we show that if $B(v, v)=0, \forall v \in \mathrm{C}$, then $B \equiv 0$, which gives a contradiction (since $B$ is non-zero). Since $v_{i}, v \in \mathrm{C}$, using the bilinearity of $B$ and (2.6), we get

$$
B\left(v_{i}-v, v_{j}-v\right)=0, \forall 1 \leq i, j \leq n,
$$

i.e., $B \equiv 0$. Therefore, $\exists v \in \mathrm{C}$ such that $B(v, v) \neq 0$.

Let $v \in \mathrm{C}$ be an element for which $B(v, v) \neq 0$. Let $\mathrm{L}_{v}=\{u \in \mathrm{V} \mid B(u, v)=0\}$. Since $B(v, v) \neq 0, V=\mathbb{R} v \oplus \mathrm{L}_{v}$. Now take $\mathrm{D}=\mathbb{R}_{>0} v \subset \mathrm{C}$, a half line contained in C. We have a basis $\left\{v, u_{1}, u_{2}, \ldots, u_{n-1}\right\}$ of $\mathrm{V}$ over $\mathbb{R}$, where $\left\{u_{1}, u_{2}, \ldots, u_{n-1}\right\}$ is a basis of $\mathrm{L}_{v}$ over $\mathbb{R}$. With respect to this basis of $\mathrm{V}, B=B_{1} \oplus B_{2}$, where $B_{1}=\left.B\right|_{\mathbb{R} v}$ and $B_{2}=\left.B\right|_{\mathrm{L}_{v}}$. The symmetric matrix associated to the bilinear form $B$, with respect to this basis, is of the form

$$
B=\left(\begin{array}{ccccc}
B_{1}(v, v) & 0 & 0 & \ldots & 0 \\
0 & & & & \\
0 & B_{2} & & & \\
\vdots & & & & \\
0 & & & &
\end{array}\right) .
$$

The group $\mathrm{G}=\mathrm{O}(B)(\mathbb{R}) \leq \mathrm{GL}(n, \mathbb{R})$, is unimodular with a Haar measure $\mu$ and it contains the Coxeter group $\mathrm{W}$ as a discrete subgroup. Let $\nu$ be a G-invariant measure on the quotient $\mathrm{W} \backslash \mathrm{G}$ such that $\nu(\mathrm{W} \backslash \mathrm{G})<\infty$, i.e., $\mathrm{W}$ is a lattice in $\mathrm{G}$.

Let $\mathrm{H}=\mathrm{O}\left(B_{2}\right)(\mathbb{R}) \leq \mathrm{GL}\left(\mathrm{L}_{v}\right)$ be the orthogonal group of the bilinear form $B_{2}$ on $\mathrm{L}_{v}$. It is clear that

$$
\mathrm{G}^{\prime}=\left\{\left(\begin{array}{cccc}
1 & 0 & \ldots & 0 \\
0 & & & \\
\vdots & & h &
\end{array}\right): h \in \mathrm{H}\right\}
$$

is a closed subgroup of $\mathrm{G}$ and $\forall g \in \mathrm{G}^{\prime}, g(v)=v$, i.e., $\mathrm{G}^{\prime}$ is a closed subgroup of $\mathrm{G}_{\mathrm{D}}$, therefore it is compact (by Lemma 2.4).

Also, $\mathrm{G}^{\prime}$ is isomorphic (as a Lie group) to $\mathrm{H}=\mathrm{O}\left(B_{2}\right)(\mathbb{R})$, therefore $\mathrm{H}$ is a compact subgroup of $\mathrm{GL}\left(\mathrm{L}_{v}\right)$. It shows that the bilinear form $B_{2}$ is either positive definite or negative definite. Since the group $\mathrm{W}$ is infinite, the bilinear form $B$ cannot be positive or negative definite. Therefore, $B$ has the signature $(n-1,1)$ or $(1, n-1)$.

Now we show that $B$ can not have the signature $(1, n-1)$.

Lemma 2.6. If there is a relation $\left(s_{i} s_{j}\right)^{m_{i, j}}=1$, for some $i \neq j$ and $2 \leq m_{i, j}<\infty$ in the generators of the Coxeter group $\mathrm{W}$ and the bilinear form $B$ as above, then $B$ has the signature $(n-1,1)$.

Proof. For $2 \leq m_{i, j}<\infty, B\left(e_{i}, e_{j}\right)=-\cos \left(\frac{\pi}{m_{i, j}}\right)>-1$, and hence

$$
\begin{aligned}
B\left(\lambda e_{i}+\delta e_{j}, \lambda e_{i}+\delta e_{j}\right) & =\lambda^{2} B\left(e_{i}, e_{i}\right)+\delta^{2} B\left(e_{j}, e_{j}\right)+2 \lambda \delta B\left(e_{i}, e_{j}\right) \\
& =\lambda^{2}+\delta^{2}+2 \lambda \delta B\left(e_{i}, e_{j}\right) \\
& >\lambda^{2}+\delta^{2}-2 \lambda \delta=(\lambda-\delta)^{2} \geq 0
\end{aligned}
$$

(since $\left.B\left(e_{i}, e_{j}\right)>-1\right)$. Therefore, $\forall \lambda, \delta \in \mathbb{R}$ and $(\lambda, \delta) \neq(0,0), B\left(\lambda e_{i}+\delta e_{j}, \lambda e_{i}\right.$ $\left.+\delta e_{j}\right)>0$. 
Let $\mathrm{V}_{i, j}=\mathbb{R} e_{i} \oplus \mathbb{R} e_{j}$ be a subspace of $\mathrm{V}$. The restriction of the bilinear form $B$ on $\mathrm{V}_{i, j}$ is non-degenerate and positive definite. Therefore, $\mathrm{V}=\mathrm{V}_{i, j} \oplus \mathrm{V}_{i, j}^{\perp}$, and with respect to a basis of $\mathrm{V}$ which is the union of a basis of $\mathrm{V}_{i, j}$ and a basis of $\mathrm{V}_{i, j}^{\perp}$, the matrix of the bilinear form $B$ is

$$
\left(\begin{array}{ccccc}
1 & 0 & 0 & \cdots & 0 \\
0 & 1 & 0 & \cdots & 0 \\
0 & 0 & & & \\
\vdots & \vdots & & \left.B\right|_{\mathrm{V}_{i, j}^{\perp}} & \\
0 & 0 & & &
\end{array}\right)
$$

and $\left.B\right|_{\mathrm{V}_{i, j}^{\perp}}$ is non-degenerate.

The above matrix form of the bilinear form $B$ shows that its signature is $(p, q)$, where $p, q \in \mathbb{N}, p+q=n$, and $p \geq 2$. Therefore, the possibility for the signature of $B$ to be $(1, n-1)$ is excluded, i.e., $B$ has the signature $(n-1,1)$.

Lemma 2.7. If $\left(s_{i} s_{j}\right)^{\infty}=1$, for $i \neq j$ and $s_{i} s_{i}=1, \forall i, j \in\{1,2, \ldots, n\}$ are the only relations in the generators of the Coxeter group $\mathrm{W}$ and the bilinear form $B$ as above, then $B$ has the signature $(n-1,1)$.

Proof. These relations mean that all the vertices in the Coxeter graph of the Coxeter group $\mathrm{W}$ are joined by an edge of weight $\infty$, and $B\left(e_{i}, e_{i}\right)=1$, and $B\left(e_{i}, e_{j}\right)=-1$, for $i \neq j$. These relations are not possible in a Coxeter group $\mathrm{W}$ with two generators $(\because B$ is non-degenerate), therefore to have the possibility stated in the statement of the lemma, $n$ must be $\geq 3$.

Since all the vertices are joined by an edge in the Coxeter graph, the Coxeter graph contains a triangle. Let $s_{1}, s_{2}$ and $s_{3}$ be any three vertices, which are joined to each other to form a triangle. Let $\mathrm{V}_{1}=\mathbb{R} e_{1} \oplus \mathbb{R} e_{2} \oplus \mathbb{R} e_{3}$ be a subspace of $\mathrm{V}$, and $B_{1}=\left.B\right|_{\mathrm{V}_{1}}$ be a bilinear form on $\mathrm{V}_{1}$. Now we show that $B_{1}$ has the signature $(2,1)$, which shows that $\mathrm{V}=\mathrm{V}_{1} \oplus \mathrm{V}_{1}^{\perp}$ and hence the signature of $B$ is $(p, q)$ with $p \geq 2$.

The matrix form of $B_{1}$ with respect to the basis $\left\{e_{1}, e_{2}, e_{3}\right\}$ of $\mathrm{V}_{1}$ over $\mathbb{R}$ is

$$
B_{1}=\left(\begin{array}{ccc}
1 & -1 & -1 \\
-1 & 1 & -1 \\
-1 & -1 & 1
\end{array}\right) .
$$

One can check easily that $2,2,-1$ are the eigenvalues of the matrix $B_{1}$.

Since a symmetric matrix is orthogonally diagonalizable, the signature of the bilinear form $B_{1}$ is $(2,1)$. It shows that the possibility for the signature of the bilinear form $B$ to be $(1, n-1)$ is excluded. Therefore, the signature of the bilinear form $B$ is $(n-1,1)$.

Since we had $\mathrm{V}=\mathbb{R} v \oplus \mathrm{L}_{v}$, where $v \in \mathrm{C}$ is an element for which $B(v, v) \neq 0$, and $\mathrm{L}_{v}=\{u \in \mathrm{V} \mid B(u, v)=0\}$, the condition on the signature of $B$ forces $B(v, v)<0$ (since $\left.B\right|_{\mathrm{L}_{v}}$ is positive definite and $B$ is non-degenerate and non-positive). The above proof also shows that if $B(u, u) \neq 0$, then $B(u, u)<0$, for any $u \in \mathrm{C}$.

Now we show that $B(u, u) \neq 0$, for any $u \in \mathrm{C}$. Otherwise, $\exists u \in \mathrm{C}$ such that $B(u, u)=0$. Since the bilinear form $B$ is non-degenerate, $\exists u^{\prime} \in \mathrm{V}$ such that $B\left(u^{\prime}, u^{\prime}\right)=$ 0 and $B\left(u, u^{\prime}\right)=1$ (see [5, Theorem 6.10]). Also, for any $\alpha, \beta>0$ in $\mathbb{R}, B(\alpha u+$ $\left.\beta u^{\prime}, \alpha u+\beta u^{\prime}\right)=2 \alpha \beta>0$. Since $u \in \mathrm{C}$, and $\mathrm{C}$ is open in $\mathrm{V}, \exists \alpha, \beta>0$ in $\mathbb{R}$ such 
that $\alpha u+\beta u^{\prime} \in \mathrm{C}$ and $B\left(\alpha u+\beta u^{\prime}, \alpha u+\beta u^{\prime}\right)=2 \alpha \beta>0$, which is a contradiction. Therefore, $B(u, u) \neq 0, \forall u \in \mathrm{C}$. Hence, $B(u, u)<0, \forall u \in \mathrm{C}$; and it completes the proof of Theorem 1.3.

\section{Proof of Theorem 1.1}

Let $\mathrm{O}(B)$ be the orthogonal group of the bilinear form $B$ and $\mathrm{O}(p, q)$ be the group of real points of the group $\mathrm{O}(B)$, i.e., $\mathrm{O}(p, q)=\mathrm{O}(B)(\mathbb{R})$, where $(p, q)$ is the signature of $B$ with $p, q \geq 1$, and $p+q=n$. Let $\mathrm{SO}(B)$ be the connected component of the identity element of $\mathrm{O}(B)$, and $\mathrm{SO}(p, q)=\mathrm{SO}(B)(\mathbb{R})$. The subgroup $\mathrm{SO}(p, q)$ has finite index (four) in the group $\mathrm{O}(p, q)$, therefore any finite index subgroup $\mathrm{L}^{\prime}$ of the Coxeter group W contains a finite index subgroup $\mathrm{L} \leq \mathrm{SO}(p, q)$, namely $\mathrm{L}=\mathrm{L}^{\prime} \cap \mathrm{SO}(p, q)$. If $\mathrm{L}^{\prime}$ is isomorphic to an irreducible lattice $\Gamma^{\prime}$ in a semisimple group $H$ of $\mathbb{R}$-rank $\geq 2$, then $\mathrm{L}$ will be isomorphic to a finite index subgroup $\Gamma$ of $\Gamma^{\prime}$. Also, it can be shown easily that a finite index subgroup $\Gamma$ of an irreducible lattice $\Gamma^{\prime}$ is an irreducible lattice in H. We prove some lemmas that will be used in the proof of Theorem 1.1.

Lemma 3.1. There exists a connected semisimple adjoint group $\tilde{\mathrm{G}}$ and an (central) isogeny $\pi: \mathrm{SO}(B) \longrightarrow \tilde{\mathrm{G}}$.

For a proof, see [7, Theorem 2.6].

In fact, $\tilde{\mathrm{G}}$ is an $\mathbb{R}$-simple group (since the group $\mathrm{SO}(B)$ has maximal normal subgroup $\{ \pm \mathrm{I}\}$ which is the center of $\mathrm{SO}(B)$ and $\pi$ is central therefore the kernel of $\pi$ is $\{ \pm \mathrm{I}\})$.

Lemma 3.2. If $\mathrm{L}$ is a discrete subgroup of $\mathrm{SO}(B)(\mathbb{R})=\mathrm{SO}(p, q)$, then $\pi(\mathrm{L})$ is a discrete subgroup of $\tilde{\mathrm{G}}(\mathbb{R})$.

Proof. The homomorphism $\pi$ is an open map and its kernel is finite. Now using the discreteness of L, it can be shown easily that $\pi(\mathrm{L})$ is a discrete subgroup of $\tilde{G}(\mathbb{R})$.

Lemma 3.3. If $\mathrm{L}$ is a Zariski dense subgroup of $\mathrm{SO}(B)$, then $\pi(\mathrm{L})$ is a Zariski dense subgroup of $\tilde{\mathrm{G}}$.

Proof. Since the map $\pi: \mathrm{SO}(B) \longrightarrow \tilde{\mathrm{G}}$ is continuous with respect to the Zariski topology, we get $\pi(\overline{\mathrm{L}}) \subseteq \overline{\pi(\mathrm{L})}$. Therefore $\overline{\pi(\mathrm{L})}=\tilde{\mathrm{G}}$ (since $\overline{\mathrm{L}}=\mathrm{SO}(B)$ ).

Lemma 3.4. $\mathbb{R}-\operatorname{rank}(\mathrm{SO}(B))=\mathbb{R}-\operatorname{rank}(\tilde{\mathrm{G}})$.

Proof. We will show that if $\mathrm{T}$ is an $\mathbb{R}$-split torus in $\mathrm{SO}(B)$, then $\pi(\mathrm{T})$ is an $\mathbb{R}$-split torus in $\tilde{\mathrm{G}}$. For, let $\mathrm{T}$ be an $\mathbb{R}$-split torus in $\mathrm{SO}(B)$, i.e., all the characters $\chi: \mathrm{T} \longrightarrow \mathbb{G}_{m}$ are defined over $\mathbb{R}$. It is clear that $\pi(\mathrm{T})$ is a connected, abelian subgroup of $\tilde{\mathrm{G}}$. Also, $\pi(\mathrm{T})$ is diagonalizable over $\mathbb{C}$ (since under a homomorphism of algebraic groups, torus maps to a torus).

To show that $\pi(\mathrm{T})$ is $\mathbb{R}$-split, it is enough to show that all the characters $\chi$ : $\pi(\mathrm{T}) \longrightarrow \mathbb{G}_{m}$ are defined over $\mathbb{R}$. For, let us define $\chi^{\prime}: \mathrm{T} \longrightarrow \mathbb{G}_{m}$ as $\chi^{\prime}(t)=\chi(\pi(t))$. It is clear that $\chi^{\prime}$ is a character of the torus $\mathrm{T}$ which is $\mathbb{R}$-split, therefore $\chi^{\prime}$ is defined over $\mathbb{R}$. Now we show that $\chi$ is fixed under the action of $\operatorname{Gal}(\mathbb{C} / \mathbb{R})$ on $\operatorname{Hom}\left(T, \mathbb{G}_{m}\right)$. 
For, let $\sigma \in \operatorname{Gal}(\mathbb{C} / \mathbb{R})$. We have

$$
\begin{aligned}
\chi(\pi(t)) & =\chi^{\prime}(t) \\
& =\left(\sigma \cdot \chi^{\prime}\right)(t) \\
& =\sigma\left(\chi^{\prime}\left(\sigma^{-1} t\right)\right) \\
& =\sigma\left(\chi \circ \pi\left(\sigma^{-1} t\right)\right) \\
& =\sigma\left(\chi \sigma^{-1}(\sigma \cdot \pi)(t)\right) \\
& =(\sigma \cdot \chi)(\pi(t))
\end{aligned}
$$$$
\left(\because \chi^{\prime} \text { and } \pi \text { are defined over } \mathbb{R}\right) \text {. }
$$

Since the above equality is true for all $t \in \mathrm{T}$ and $\pi$ is surjective, therefore we get $\sigma \cdot \chi=\chi$, for all $\sigma \in \operatorname{Gal}(\mathbb{C} / \mathbb{R})$. Hence all the characters $\chi: \pi(\mathrm{T}) \longrightarrow \mathbb{G}_{m}$ are defined over $\mathbb{R}$, i.e., $\pi(\mathrm{T})$ is an $\mathbb{R}$-split torus in $\tilde{\mathrm{G}}$. Since $\pi$ has finite kernel, we get $\mathbb{R}-\operatorname{rank}(\tilde{\mathrm{G}})$ $=\mathbb{R}-\operatorname{rank}(\mathrm{SO}(B))$.

Theorem 3.5. Let $\mathrm{L}$ be a discrete subgroup of the group $\mathrm{SO}(p, q)$. Let $\mathrm{H}$ be a connected semisimple Lie group without non-trivial compact factor groups, of real rank $\geq 2$ with trivial center. Let $\Gamma \leq \mathrm{H}$ be an irreducible lattice and $\delta: \Gamma \longrightarrow \mathrm{L} \leq$ $\mathrm{SO}(B)(\mathbb{R})=\mathrm{SO}(p, q)$ be an isomorphism and $\delta(\Gamma)=\mathrm{L}$ is Zariski dense in $\mathrm{SO}(B)$. Let $\tilde{\mathrm{G}}$ be a connected semisimple adjoint group with an (central) isogeny $\pi: \mathrm{SO}(B) \longrightarrow \tilde{\mathrm{G}}$. Let $\delta^{\prime}: \Gamma \longrightarrow \pi(\mathrm{L}) \leq \tilde{\mathrm{G}}(\mathbb{R})$ be a continuous homomorphism defined as $\delta^{\prime}=\pi \circ \delta$. Let $\tilde{\mathrm{G}}$ has no non-trivial $\mathbb{R}$-anisotropic factors and $\tilde{\mathrm{G}}(\mathbb{R})^{\circ}$ be the connected component of the identity element in $\tilde{G}(\mathbb{R})$. Then $\delta^{\prime}$ extends uniquely to an isomorphism $\tilde{\delta}^{\prime}: \mathrm{H} \longrightarrow$ $\tilde{\mathrm{G}}(\mathbb{R})^{\circ}$, and the group $\tilde{\mathrm{G}}(\mathbb{R})$ has $\mathbb{R}-$ rank $\geq 2$, and $\pi(\mathrm{L})$ is a lattice in $\tilde{\mathrm{G}}(\mathbb{R})$.

Proof. The group $\tilde{\mathrm{G}}$ is adjoint, and has no non-trivial $\mathbb{R}$-anisotropic factors and $\pi(\mathrm{L})$ is a discrete subgroup of $\tilde{\mathrm{G}}(\mathbb{R})$ (by Lemma 3.2 ), and it is also Zariski dense in $\tilde{\mathrm{G}}$ (by Lemma 3.3). Therefore by Theorem 1.4, we get a continuous homomorphism $\tilde{\delta}^{\prime}: \mathrm{H} \longrightarrow \tilde{\mathrm{G}}(\mathbb{R})$ with $\left.\tilde{\delta}^{\prime}\right|_{\Gamma}=\delta^{\prime}$. Since the group $\tilde{\delta}^{\prime}(\mathrm{H})$ is a connected semisimple group which is Zariski dense in $\tilde{\mathrm{G}}$ ( since $\tilde{\delta}^{\prime}(\Gamma)=\pi(\mathrm{L})$ is Zariski dense in $\tilde{\mathrm{G}}$ ), it follows from [6] (Remark 6.17 (ii) of Chapter IX) that $\tilde{\delta}^{\prime}(\mathrm{H})=\tilde{\mathrm{G}}(\mathbb{R})^{\circ}$. Since $\mathrm{H}$ has trivial center and no non-trivial compact factor groups, $\Gamma$ is an irreducible lattice in $\mathrm{H}$, and $\delta^{\prime}(\Gamma)=\pi(\mathrm{L})$ is a non-trivial discrete subgroup of $\tilde{\mathrm{G}}(\mathbb{R})$, therefore it follows from $[6]$ (Remark 6.17 (iii) of Chapter IX) that $\tilde{\delta}^{\prime}$ is an isomorphism of $\mathrm{H}$ onto $\tilde{\mathrm{G}}(\mathbb{R})^{\circ}$, and hence $\pi(\mathrm{L})$ is a lattice in $\tilde{\mathrm{G}}(\mathbb{R})^{\circ}$, and the $\mathbb{R}$-rank of $\tilde{\mathrm{G}}(\mathbb{R})$ is $\geq 2$. Since $\tilde{\mathrm{G}}(\mathbb{R})^{\circ}$ is a finite index subgroup of $\tilde{G}(\mathbb{R}), \pi(L)$ is a lattice in $\tilde{G}(\mathbb{R})$.

Remark. In the proof of Theorem 3.5, the fact that $\mathrm{H}$ has trivial center, has been used only to show that $\tilde{\delta}^{\prime}$ is an isomorphism. If the group $\mathrm{H}$ does not have trivial center, then the homomorphism $\tilde{\delta}^{\prime}$ has finite kernel, and $\tilde{\delta}^{\prime}(\Gamma)=\pi(\mathrm{L})$ is still a lattice in $\tilde{G}(\mathbb{R})$ (since under such homomorphism $\tilde{\delta}^{\prime}$, a lattice maps onto a lattice). Therefore Theorem 3.5 is also true for a connected semisimple Lie group with non-trivial center, and without non-trivial compact factor groups, of real rank $\geq 2$.

Lemma 3.6. Let $\mathrm{L}$ be a discrete subgroup of $\mathrm{SO}(p, q)$, and $\tilde{\mathrm{G}}, \pi$ as in Lemma 3.1. If $\pi(\mathrm{L})$ is a lattice in $\tilde{\mathrm{G}}(\mathbb{R})$, then $\mathrm{L}$ is a lattice in $\mathrm{SO}(p, q)$. 
Proof. Since L is a discrete subgroup of $\mathrm{SO}(p, q)$ and $\mathrm{SO}(p, q)$ is unimodular, the quotient $\mathrm{L} \backslash \mathrm{SO}(p, q)$ has an $\mathrm{SO}(p, q)$-invariant measure $\mu$. The homomorphism $\pi$ : $\mathrm{SO}(p, q) \longrightarrow \tilde{\mathrm{G}}(\mathbb{R})$ induces a continuous map $\tilde{\pi}: \mathrm{L} \backslash \mathrm{SO}(p, q) \longrightarrow \pi(\mathrm{L}) \backslash \tilde{\mathrm{G}}(\mathbb{R})$, which is defined as $\tilde{\pi}(\mathrm{L} g)=\pi(\mathrm{L}) \pi(g)$. It can be checked easily that the pushforward measure $\tilde{\pi}_{*}(\mu)$ on the quotient $\pi(\mathrm{L}) \backslash \tilde{\mathrm{G}}(\mathbb{R})$ defined as $\tilde{\pi}_{*}(\mu)(\tilde{\mathrm{E}})=\mu\left(\tilde{\pi}^{-1}(\tilde{\mathrm{E}})\right)$, for all measurable subsets $\tilde{\mathrm{E}}$ of $\pi(\mathrm{L}) \backslash \tilde{\mathrm{G}}(\mathbb{R})$, is $\tilde{\mathrm{G}}(\mathbb{R}$ )-invariant (since $\tilde{\pi}$ is surjective and $\mu$ is $\operatorname{SO}(p, q)$ invariant). Therefore by the uniqueness of a $\tilde{G}(\mathbb{R})$-invariant measure on the quotient $\pi(\mathrm{L}) \backslash \tilde{\mathrm{G}}(\mathbb{R})$, we get $\tilde{\pi}_{*}(\mu)(\pi(\mathrm{L}) \backslash \tilde{\mathrm{G}}(\mathbb{R}))<\infty$ (since $\pi(\mathrm{L})$ is a lattice in $\tilde{\mathrm{G}}(\mathbb{R})$ ), and hence $\mu(\mathrm{L} \backslash \mathrm{SO}(p, q))<\infty$, i.e., $\mathrm{L}$ is a lattice in $\mathrm{SO}(p, q)$.

Theorem 3.7. The Coxeter group $\mathrm{W}$ is Zariski dense in the group $\mathrm{O}(B)$.

For a proof, see [1].

Lemma 3.8. Let $\mathrm{G}$ be a topological group and $\mathrm{L}^{\prime}, \mathrm{L}$ are subgroups of $\mathrm{G}$ such that $\mathrm{L}$ has finite index in $\mathrm{L}^{\prime}$. Then $\left(\overline{\mathrm{L}}^{\prime}\right)^{\mathrm{O}}=(\overline{\mathrm{L}})^{\mathrm{o}}$, where $(\overline{\mathrm{L}})^{\mathrm{o}}$ is the connected component of the identity element of the closure of $\mathrm{L}$ in $\mathrm{G}$.

Proof. Since L has finite index $d$ (say) in $\mathrm{L}^{\prime}$,

$$
\begin{aligned}
\mathrm{L}^{\prime} & =\cup_{i=1}^{d} \gamma_{i} \mathrm{~L} ; \gamma_{i} \in \mathrm{L}^{\prime} \\
& \Rightarrow \overline{\mathrm{L}^{\prime}}=\cup_{i=1}^{d} \gamma_{i} \overline{\mathrm{L}} ; \gamma_{i} \in \mathrm{L}^{\prime} \\
& \Rightarrow\left[\overline{\mathrm{L}^{\prime}}: \overline{\mathrm{L}}\right] \leq d \\
& \Rightarrow \overline{\mathrm{L}} \text { is a finite index subgroup of the group } \overline{\mathrm{L}^{\prime}} .
\end{aligned}
$$

Hence $\overline{\mathrm{L}}$ is closed and open in $\overline{\mathrm{L}}^{\prime}$ and $\left(\overline{\mathrm{L}}^{\prime}\right)^{\mathrm{o}} \supset(\overline{\mathrm{L}})^{\mathrm{o}}$, therefore $(\overline{\mathrm{L}})^{\circ}$ is open and closed in $\left(\overline{\mathrm{L}^{\prime}}\right)^{\mathrm{o}}$ which is connected. This shows that $\left(\overline{\mathrm{L}^{\prime}}\right)^{\mathrm{o}}=(\overline{\mathrm{L}})^{\mathrm{o}}$.

Corollary 3.9. In the above lemma if we take $\mathrm{G}=\mathrm{O}(p, q)=\mathrm{O}(B)(\mathbb{R})$, and $\mathrm{L}^{\prime}=\mathrm{W}$, the Coxeter group and $\mathrm{L} \leq \mathrm{SO}(p, q) \cap \mathrm{W}$ such that $[\mathrm{W}: \mathrm{L}]<\infty$, then $\overline{\mathrm{L}}=\mathrm{SO}(p, q)$, i.e., $\mathrm{L}$ is Zariski dense in $\mathrm{SO}(p, q)$. Hence $\mathrm{L}$ is Zariski dense in $\mathrm{SO}(B)(\because \mathrm{SO}(B)(\mathbb{R})=$ $\mathrm{SO}(p, q)$ is Zariski dense in $\mathrm{SO}(B))$.

Proof. The proof follows from Theorem 3.7 and Lemma 3.8 .

Lemma 3.10. If $\mathrm{L}$ is a lattice in $\mathrm{SO}(p, q)$, then $\mathrm{L}$ is also a lattice in $\mathrm{O}(p, q)$.

Proof. Since $\mathrm{O}(p, q)$ is unimodular and $\mathrm{L}$ is a discrete subgroup of $\mathrm{O}(p, q)$, we get $\mathrm{L} \backslash \mathrm{O}(p, q)$ has a non-zero $\mathrm{O}(p, q)$-invariant measure $\mu$. Since $\mathrm{SO}(p, q)$ is open in $\mathrm{O}(p, q)$, its Borel $\sigma$-algebra is a subalgebra of the Borel $\sigma$-algebra of $\mathrm{O}(p, q)$ and the restriction of $\mu$ on $\mathrm{L} \backslash \mathrm{SO}(p, q)$ is a non-zero $\mathrm{SO}(p, q)$-invariant measure. Now we claim that $\mu(\mathrm{L} \backslash \mathrm{O}(p, q))<\infty$. For,

$$
\mathrm{L} \backslash \mathrm{O}(p, q)=\{\mathrm{L} g \mid g \in \mathrm{O}(p, q)\}
$$

and

$$
\mathrm{O}(p, q)=\left\{\mathrm{SO}(p, q) g_{i} \mid g_{i} \in \mathrm{O}(p, q), 1 \leq i \leq 4\right\}
$$


i.e., $\forall g \in \mathrm{O}(p, q), \exists h \in \mathrm{SO}(p, q)$ such that $g=h g_{i}$, for some $1 \leq i \leq 4$. Therefore, $\mathrm{L} g=\mathrm{Lh}_{i} \in(\mathrm{L} \backslash \mathrm{SO}(p, q)) g_{i}$, and

$$
\begin{aligned}
\mathrm{L} \backslash \mathrm{O}(p, q) & =\cup_{i=1}^{4}(\mathrm{~L} \backslash \mathrm{SO}(p, q)) g_{i} . \\
\Rightarrow \mu(\mathrm{L} \backslash \mathrm{O}(p, q)) & \leq \sum_{i=1}^{4} \mu\left((\mathrm{L} \backslash \mathrm{SO}(p, q)) g_{i}\right) \\
& =\sum_{i=1}^{4} \mu(\mathrm{L} \backslash \mathrm{SO}(p, q)) \\
& <\infty .
\end{aligned}
$$

It shows that $\mathrm{L}$ is a lattice in $\mathrm{O}(p, q)$.

From the remark at the beginning of this section and Corollary 3.9, it follows that if the Coxeter group $\mathrm{W}$ contains a finite index subgroup $\mathrm{L} \leq \mathrm{SO}(p, q)$, which is isomorphic to an irreducible lattice in a connected semisimple Lie group $\mathrm{H}$ without non-trivial compact factor groups, of real rank $\geq 2$, then $\mathrm{SO}(p, q)$ has real rank $\geq 2$ (by Lemma 3.4 and Theorem 3.5), i.e., $p, q \geq 2$, and $\mathrm{L}$ is a lattice in $\mathrm{SO}(p, q)$ (by Theorem 3.5 and Lemma 3.6). Moreover, Lemma 3.10 shows that L is a lattice in $\mathrm{O}(p, q)$ also, and hence $\mathrm{W}$ becomes a lattice in $\mathrm{O}(p, q)$ (since a discrete subgroup $\mathrm{W}$ of a Lie group $\mathrm{G}$ which contains a lattice $\mathrm{L}$, is a lattice in $\mathrm{G}$ ). This is a contradiction to Theorem 1.3, which has been proved in Section 2; and it completes the proof of Theorem 1.1.

\section{Right-angled Coxeter group with three generators}

In this section, we will do some computations and show that a right-angled Coxeter group $\mathrm{W}$ generated by three elements is isomorphic to a lattice in the group $\mathrm{O}(B)(\mathbb{R})=\mathrm{O}(2,1)$ of real rank 1 .

Let $\mathrm{W}$ be the right-angled Coxeter group generated by three elements $s_{1}, s_{2}$ and $s_{3}$ with the relations: $\left(s_{i} s_{j}\right)^{m_{i, j}}=1$, where $m_{i, i}=1, \forall i \in\{1,2,3\}$, and $m_{1,2}=m_{2,3}=\infty$, $m_{1,3}=2$. Let $\mathbb{R}^{3}$ be a three-dimensional vector space over $\mathbb{R}$ with a basis $\left\{e_{1}, e_{2}, e_{3}\right\}$. We define a symmetric bilinear form $B$ on $\mathbb{R}^{3}$ as

$$
B\left(e_{i}, e_{j}\right)=-\cos \left(\frac{\pi}{m_{i, j}}\right), \text { for } m_{i, j} \neq \infty,
$$

and for $m_{i, j}=\infty$, we define $B\left(e_{i}, e_{j}\right)=-1$. With respect to the basis $\left\{e_{1}, e_{2}, e_{3}\right\}$, the matrix of $B$ is

$$
B=\left(\begin{array}{ccc}
1 & -1 & 0 \\
-1 & 1 & -1 \\
0 & -1 & 1
\end{array}\right)
$$

One can check that the bilinear form $B$ is non-degenerate.

Now we define a representation $\rho: \mathrm{W} \longrightarrow \mathrm{GL}\left(\mathbb{R}^{3}\right)$ by defining $\rho\left(s_{i}\right)\left(e_{j}\right)=e_{j}-$ $2 B\left(e_{j}, e_{i}\right) e_{i}$, which is faithful (by [4, Corollary 5.4]). It can be checked easily that $\rho$ maps the group $\mathrm{W}$ inside the orthogonal group $\mathrm{O}(B)(\mathbb{R})$ of the bilinear form $B$. We will show that the group $\mathrm{W}$ is mapped (by $\rho$ ) onto a finite index subgroup of $\mathrm{O}(B)(\mathbb{Z})$, the group of integral points of the orthogonal group $\mathrm{O}(B)$, and it shows that the group $\mathrm{W}$ is a lattice in $\mathrm{O}(B)(\mathbb{R})$. 
With respect to the basis $\left\{e_{1}, e_{2}, e_{3}\right\}$, the matrices of $\rho\left(s_{1}\right), \rho\left(s_{2}\right)$ and $\rho\left(s_{3}\right)$ are

$$
\rho\left(s_{1}\right)=\left(\begin{array}{ccc}
-1 & 2 & 0 \\
0 & 1 & 0 \\
0 & 0 & 1
\end{array}\right), \quad \rho\left(s_{2}\right)=\left(\begin{array}{ccc}
1 & 0 & 0 \\
2 & -1 & 2 \\
0 & 0 & 1
\end{array}\right), \quad \rho\left(s_{3}\right)=\left(\begin{array}{ccc}
1 & 0 & 0 \\
0 & 1 & 0 \\
0 & 2 & -1
\end{array}\right) .
$$

If we do some integral change in the basis of $\mathbb{R}^{3}$ over $\mathbb{R}$, and take $\left\{e_{1}+e_{2}, e_{2}, e_{2}+e_{3}\right\}$ as a basis of $\mathbb{R}^{3}$, then the corresponding matrices of $\rho\left(s_{1}\right), \rho\left(s_{2}\right), \rho\left(s_{3}\right)$ and $B$, become

$$
\rho\left(s_{1}\right)=\left(\begin{array}{ccc}
1 & 2 & 2 \\
0 & -1 & -2 \\
0 & 0 & 1
\end{array}\right), \quad \rho\left(s_{2}\right)=\left(\begin{array}{ccc}
1 & 0 & 0 \\
0 & -1 & 0 \\
0 & 0 & 1
\end{array}\right), \quad \rho\left(s_{3}\right)=\left(\begin{array}{ccc}
1 & 0 & 0 \\
-2 & -1 & 0 \\
2 & 2 & 1
\end{array}\right)
$$

and

$$
B=\left(\begin{array}{ccc}
0 & 0 & -1 \\
0 & 1 & 0 \\
-1 & 0 & 0
\end{array}\right)
$$

It is now clear that the signature of the bilinear form $B$ is $(2,1)$.

The adjoint representation of $\mathrm{SL}(2, \mathbb{R})$ on its Lie algebra $\operatorname{sl}(2, \mathbb{R})$, maps the group $\operatorname{PSL}(2, \mathbb{R})=\operatorname{SL}(2, \mathbb{R}) /\{ \pm \mathrm{I}\}$ isomorphically onto its image and it preserves the killing form $K$ defined on $\operatorname{sl}(2, \mathbb{R})$. The Lie algebra $\operatorname{sl}(2, \mathbb{R})$ can be identified with $\mathbb{R}^{3}$ as a vector space over $\mathbb{R}$, with the basis

$$
\left\{e_{1}=\left(\begin{array}{ll}
0 & 1 \\
0 & 0
\end{array}\right), e_{2}=\left(\begin{array}{cc}
1 & 0 \\
0 & -1
\end{array}\right), e_{3}=\left(\begin{array}{ll}
0 & 0 \\
1 & 0
\end{array}\right)\right\} \text {. }
$$

The killing form $K$ on $\mathrm{sl}(2, \mathbb{R}))$ is defined by

$$
K(X, Y)=\frac{1}{2} \operatorname{tr}(X Y), \quad \forall X, Y \in \operatorname{sl}(2, \mathbb{R}) .
$$

If we do some integral change in the basis of $\operatorname{sl}(2, \mathbb{R})$ over $\mathbb{R}$ and take

$$
\left\{\epsilon_{1}=-2 e_{1}=\left(\begin{array}{cc}
0 & -2 \\
0 & 0
\end{array}\right), \epsilon_{2}=e_{2}=\left(\begin{array}{cc}
1 & 0 \\
0 & -1
\end{array}\right), \epsilon_{3}=e_{3}=\left(\begin{array}{ll}
0 & 0 \\
1 & 0
\end{array}\right)\right\}
$$

as a basis of $\operatorname{sl}(2, \mathbb{R})$ over $\mathbb{R}$, then the matrix of $K$ becomes

$$
K=\left(\begin{array}{ccc}
0 & 0 & -1 \\
0 & 1 & 0 \\
-1 & 0 & 0
\end{array}\right)
$$

Therefore, the bilinear form $B$ associated to the Coxeter group $\mathrm{W}$, is equivalent to the killing form $K$ on $\operatorname{sl}(2, \mathbb{R})$ over $\mathbb{Z}$, and the signature of $K$ is also $(2,1)$. Hence the group $\mathrm{SL}(2, \mathbb{R}) /\{ \pm \mathrm{I}\}$ maps into $\mathrm{O}(2,1) \leq \mathrm{GL}(3, \mathbb{R})$, by the adjoint representation Ad of $\mathrm{SL}(2, \mathbb{R})$ on its Lie algebra, where $\mathrm{O}(2,1)=\mathrm{O}(B)(\mathbb{R})$. Since the group $\mathrm{SL}(2, \mathbb{R}) /\{ \pm \mathrm{I}\}$ is connected, it is mapped inside $\mathrm{SO}(2,1)$, the connected component of the identity element in $\mathrm{O}(2,1)$. In fact, $\operatorname{Ad}(\mathrm{SL}(2, \mathbb{R}) /\{ \pm \mathrm{I}\})=\mathrm{SO}(2,1)(\because \operatorname{dim} \mathrm{SL}(2, \mathbb{R}) /\{ \pm \mathrm{I}\}=\operatorname{dim}$ $\mathrm{SO}(2,1))$, i.e., $\mathrm{SL}(2, \mathbb{R}) /\{ \pm \mathrm{I}\} \cong \mathrm{SO}(2,1)$. Hence $\mathrm{SL}(2, \mathbb{Z}) /\{ \pm \mathrm{I}\}$ is a lattice in $\mathrm{SO}(2,1)$. In fact, $\mathrm{SL}(2, \mathbb{Z}) /\{ \pm \mathrm{I}\}$ is a lattice in $\mathrm{O}(2,1)(\because \mathrm{SO}(2,1)$ has finite index in $\mathrm{O}(2,1))$.

The right-angled Coxeter group $\mathrm{W}$ is mapped inside $\mathrm{O}(B)(\mathbb{Z})=\mathrm{O}(2,1)(\mathbb{Z})$, by the representation $\rho$. We construct a finite index subgroup $\mathrm{H}$ of $\mathrm{SL}(2, \mathbb{Z}) /\{ \pm \mathrm{I}\}$ which preserves a lattice $\mathrm{L}$ in $\operatorname{sl}(2, \mathbb{R})=\mathbb{R}^{3}$ (as a vector space), i.e., $\mathrm{H}$ is also mapped inside $\mathrm{O}(2,1)(\mathbb{Z})$, by the representation $\mathrm{Ad}$, and being a finite index subgroup of 
$\mathrm{SL}(2, \mathbb{Z}) /\{ \pm \mathrm{I}\}, \mathrm{H}$ becomes a lattice in $\mathrm{O}(2,1)$. Also, we construct a finite index subgroup $\mathrm{H}^{\prime}$ of $\mathrm{W}$ which is mapped onto $\mathrm{Ad}(\mathrm{H})$, by the representation $\rho$, and hence $\rho\left(\mathrm{H}^{\prime}\right)$ becomes a lattice in $\mathrm{O}(2,1)$, and $\mathrm{W}$ becomes a finite index subgroup of $\mathrm{O}(2,1)(\mathbb{Z})$, i.e., a lattice in $\mathrm{O}(2,1)$.

Lemma 4.1. The group $\mathrm{SL}(2, \mathbb{Z}) /\{ \pm \mathrm{I}\}$ is generated by $w=\left(\begin{array}{cc}0 & -1 \\ 1 & 0\end{array}\right)$ and $x=\left(\begin{array}{ll}1 & 1 \\ 0 & 1\end{array}\right)$, and it has a presentation as $\left\langle w, x ; w^{2},(w x)^{3}\right\rangle$, i.e., it is the free product of the cyclic group of order 2 generated by $w$ and the cyclic group of order 3 generated by $w x$.

For a proof, see Theorem 2 and the preceding remark of Chapter VII in [8].

We get $x^{2}=\left(\begin{array}{ll}1 & 2 \\ 0 & 1\end{array}\right)$ and $w x^{2} w^{-1}=\left(\begin{array}{cc}1 & 0 \\ -2 & 1\end{array}\right)=\left(\begin{array}{ll}1 & 0 \\ 2 & 1\end{array}\right)^{-1}$.

Let $\mathrm{H}$ be the subgroup of $\mathrm{SL}(2, \mathbb{Z}) /\{ \pm \mathrm{I}\}$ generated by $\left\{x^{2}, w x^{2} w^{-1}\right\}$. It can be shown using the presentation of $\mathrm{SL}(2, \mathbb{Z}) /\{ \pm \mathrm{I}\}$ as in the above lemma, that the subgroup $\mathrm{H}$ has finite index in $\mathrm{SL}(2, \mathbb{Z}) /\{ \pm \mathrm{I}\}$. Also, one can show easily that $\mathrm{H}$ preserves the lattice

$$
\mathrm{L}=\mathbb{Z}\left(\begin{array}{cc}
0 & -2 \\
0 & 0
\end{array}\right) \oplus \mathbb{Z}\left(\begin{array}{cc}
1 & 0 \\
0 & -1
\end{array}\right) \oplus \mathbb{Z}\left(\begin{array}{ll}
0 & 0 \\
1 & 0
\end{array}\right)
$$

in $\operatorname{sl}(2, \mathbb{R})$. Hence $\mathrm{H}$ is mapped inside $\mathrm{O}(2,1)(\mathbb{Z})$, by the adjoint representation Ad, and being a lattice $(\because$ it has finite index in $\operatorname{SL}(2, \mathbb{Z}) /\{ \pm \mathrm{I}\})$ in $\mathrm{O}(2,1)(\mathbb{R})$, it has finite index in $\mathrm{O}(2,1)(\mathbb{Z})$. By an easy computation, we find that the matrices of $\operatorname{Ad}\left(x^{2}\right)$, $\operatorname{Ad}\left(w x^{2} w^{-1}\right)^{-1}$ in $\mathrm{O}(2,1)(\mathbb{R})$ with respect to the basis

$$
\left\{\epsilon_{1}=-2 e_{1}=\left(\begin{array}{cc}
0 & -2 \\
0 & 0
\end{array}\right), \quad \epsilon_{2}=e_{2}=\left(\begin{array}{cc}
1 & 0 \\
0 & -1
\end{array}\right), \quad \epsilon_{3}=e_{3}=\left(\begin{array}{ll}
0 & 0 \\
1 & 0
\end{array}\right)\right\}
$$

are

$$
\operatorname{Ad}\left(x^{2}\right)=\left(\begin{array}{lll}
1 & 2 & 2 \\
0 & 1 & 2 \\
0 & 0 & 1
\end{array}\right), \quad \operatorname{Ad}\left(w x^{2} w^{-1}\right)^{-1}=\left(\begin{array}{lll}
1 & 0 & 0 \\
4 & 1 & 0 \\
8 & 4 & 1
\end{array}\right) .
$$

Let $\mathrm{H}^{\prime}$ be the subgroup of the Coxeter group $\mathrm{W}$ generated by the set $\left\{s_{2} s_{1}, s_{2} s_{3}\right\}$. It can be shown easily that the subgroup $\mathrm{H}^{\prime}$ has finite index in the group W. We find that the matrices of $\rho\left(s_{2} s_{1}\right)$ and $\rho\left(s_{2} s_{3}\right)$ in $\mathrm{O}(2,1)(\mathbb{R})$ with respect to the basis $\left\{e_{1}+e_{2}, e_{2}, e_{2}+e_{3}\right\}$, are

$$
\rho\left(s_{2} s_{1}\right)=\left(\begin{array}{ccc}
1 & 2 & 2 \\
0 & 1 & 2 \\
0 & 0 & 1
\end{array}\right) \quad \text { and } \quad \rho\left(s_{2} s_{3}\right)=\left(\begin{array}{ccc}
1 & 0 & 0 \\
2 & 1 & 0 \\
2 & 2 & 1
\end{array}\right) .
$$

Also, $\rho\left(s_{2} s_{3}\right)^{2}=\left(\begin{array}{lll}1 & 0 & 0 \\ 4 & 1 & 0 \\ 8 & 4 & 1\end{array}\right)=\operatorname{Ad}\left(w x^{2} w^{-1}\right)^{-1}$, and hence by (4.1) and (4.2), we see that $\mathrm{H}$ is a subgroup of $\mathrm{H}^{\prime}$. Therefore $\mathrm{H}^{\prime}$ is a finite index subgroup of $\mathrm{O}(2,1)(\mathbb{Z})$, and hence the Coxeter group $\mathrm{W}$ is also a finite index subgroup of $\mathrm{O}(2,1)(\mathbb{Z})$, i.e., $\mathrm{W}$ is a lattice in $\mathrm{O}(2,1)$.

\section{Acknowledgments}

I sincerely thank my advisor Professor T. N. Venkataramana for suggesting me this problem and for many useful discussions. I also thank Professor James E. Humphreys for suggesting me a reference to a Bourbaki ([2]) exercise (Theorem 1.3 in this paper). 


\section{References}

[1] Y. Benoist and P. de la Harpe, Adhérence de Zariski des groupes de Coxeter, Compos. Math. 140(5) (2004), 1357-1366.

[2] N. Bourbaki, Lie groups and Lie algebras, Chapter 4-6, Elements of Mathematics, Springer-Verlag, 2002.

[3] D. Cooper, D.D. Long and A.W. Reid, Infinite Coxeter groups are virtually indicable, Proc. Edinburgh Math. Soc. 41(2) (1998), 303-313.

[4] J.E. Humphreys, Reflection groups and Coxeter groups, Cambridge Studies in Advanced Mathematics, 29, Cambridge University Press, 1990.

[5] N. Jacobson, Basic algebra. I., W. H. Freeman and Company, 1985.

[6] G.A. Margulis, Discrete Subgroups of Semisimple Lie Groups, Ergebnisse der Mathematik und ihrer Grenzgebiete (3), Springer-Verlag, 1991.

[7] V. Platonov and A. Rapinchuk, Algebraic groups and number theory, Academic Press, 1994.

[8] J.-P. Serre, A course in Arithmetic, graduate text in mathematics, Springer-Verlag, 1973.

[9] T.N. Venkataramana, On superrigidity and arithmeticity of lattices in semisimple groups over local fields of arbitrary characteristic, Invent. math. 92(2) (1988), 255-306.

School of Mathematics, Tata Institute of Fundamental Research, Homi Bhabha Road, Mumbai 400005, India

E-mail address: sandips@math.tifr.res.in 